\title{
A Modified Generalized Laguerre-Gauss Collocation Method for Fractional Neutral Functional-Differential Equations on the Half-Line
}

\author{
Ali H. Bhrawy, ${ }^{1,2}$ Abdulrahim AlZahrani, ${ }^{3}$ Dumitru Baleanu, ${ }^{3,4,5}$ and Yahia Alhamed ${ }^{3}$ \\ ${ }^{1}$ Department of Mathematics, Faculty of Science, King Abdulaziz University, Jeddah, Saudi Arabia \\ ${ }^{2}$ Department of Mathematics, Faculty of Science, Beni-Suef University, Beni Suef, Egypt \\ ${ }^{3}$ Chemical and Materials Engineering Department, Faculty of Engineering, King Abdulaziz University, Jeddah, Saudi Arabia \\ ${ }^{4}$ Department of Mathematics and Computer Sciences, Cankaya University, 06530 Ankara, Turkey \\ ${ }^{5}$ Institute of Space Sciences, Magurele, 76900 Bucharest, Romania \\ Correspondence should be addressed to Dumitru Baleanu; dumitru.baleanu@gmail.com
}

Received 22 April 2014; Accepted 8 May 2014; Published 9 June 2014

Academic Editor: Guo-Cheng Wu

Copyright (c) 2014 Ali H. Bhrawy et al. This is an open access article distributed under the Creative Commons Attribution License, which permits unrestricted use, distribution, and reproduction in any medium, provided the original work is properly cited.

\begin{abstract}
The modified generalized Laguerre-Gauss collocation (MGLC) method is applied to obtain an approximate solution of fractional neutral functional-differential equations with proportional delays on the half-line. The proposed technique is based on modified generalized Laguerre polynomials and Gauss quadrature integration of such polynomials. The main advantage of the present method is to reduce the solution of fractional neutral functional-differential equations into a system of algebraic equations. Reasonable numerical results are achieved by choosing few modified generalized Laguerre-Gauss collocation points. Numerical results demonstrate the accuracy, efficiency, and versatility of the proposed method on the half-line.
\end{abstract}

\section{Introduction}

The fractional calculus which means the calculus of derivatives and integral of any order started to be used as a powerful tool in various disciplines from science and engineering $[1$, $2]$. The models involving fractional derivatives and integrals have memory; therefore it has proven to be very suitable for the description of memory and hereditary properties of various processes [3-11]. Interested readers can also see [12-21].

Neutral functional-differential equations play an important role in the mathematical modeling of several phenomena. It is well known that most of delay differential equations cannot be solved exactly. Therefore, numerical methods would be presented and developed to get approximate solutions of these equations. The rational approximation scheme has been proposed by Ishiwata and Muroya [22], for approximating the solution of delay differential equations. Moreover, the authors of [23] investigated the variational iteration method to find a closed form analytical solution of a class of the neutral functional-differential equations. Recently, the authors of [24] proposed the Chebyshev cardinal functions in combination of operational matrices for numerical solution of delay differential equations which arise in electrodynamics. Very recently, Tohidi et al. [25] proposed and developed an efficient collocation method which depends on Bernoulli operational matrix for numerical solution of a class of delay differential equations. Meanwhile, Doha et al. [26] proposed an accurate approximate solution of generalized pantograph equations on the half-line using Jacobi rationalGauss collocation method. In this paper we propose an approximate solution of a class of delay differential equations, namely, fractional neutral functional-differential equations (FNFDEs) with proportional delay.

In the last decade or so, comprehensive research has been accomplished on the development of numerical algorithms which are numerically stable for both linear and nonlinear FDEs. Tripathi et al. [27] presented a new operational matrix of hat functions to solve linear FDEs. The spectral tau method was proposed in [28] to achieve an accurate solution of linear and nonlinear FDEs subject to multipoint conditions. In [29], 
Ma and Huang developed spectral collocation method for solving linear fractional integrodifferential equations. Yang and Huang [30] analyzed and developed the Jacobi collocation scheme for pantograph integrodifferential equations with fractional orders in finite interval. In [31], Yin et al. proposed a new fractional-order Legendre function with spectral method to solve partial FDEs; based on the operational matrix of these functions, the same authors developed their approach in combination with variational iteration formula to solve a class of FDEs; see [32]. More recently, the Jacobi Galerkin method was extended in [33] to solve stochastic FDEs, for fractional differential equations in unbounded domains. The operation matrices of fractional derivatives and fractional integrals of generalized Laguerre polynomials were investigated for solving multiterm FDEs on a semi-infinite interval; see $[11,12]$. The generalized Laguerre spectral tau and collocation techniques were given in [12] to solve linear and nonlinear FDEs on the half- line. These spectral techniques were developed and generalized by using the modified generalized Laguerre polynomials in [34-36].

Our fundamental goal of this paper is to develop a suitable way to approximate the neutral fractional functionaldifferential equations with proportional delays on the interval $(0, \infty)$ using the modified generalized Laguerre polynomials; we propose the spectral modified generalized LaguerreGauss collocation (MGLC) method to find the solution $u_{N}(x)$. The modified generalized Laguerre spectral collocation (MGLC) approximation, which is more reliable, is employed to obtain approximate solution of neutral fractional functional-differential equations with proportional delays of order $v(m-1<v<m)$ and $m$ initial conditions. For suitable collocation points we use the $(N-m+1)$ nodes of the modified generalized Laguerre-Gauss interpolation on $(0, \infty)$. These equations together with initial conditions generate $(N+1)$ algebraic equations which can be solved. Finally, the accuracy of the proposed methods is demonstrated by test problems; numerical results are presented in which the usual exponential convergence behavior of spectral approximations is exhibited.

This paper is organized as follows. In Section 2 we give an overview of modified generalized Laguerre polynomials and their relevant properties needed hereafter, and in Section 3, the way of constructing the collocation technique for neutral fractional functional-differential equations with proportional delays is described using the modified generalized Laguerre polynomials. In Section 4, we present some numerical results exhibiting the accuracy and efficiency of our numerical algorithms. The last section offers some obtained conclusions.

\section{Some Basic Preliminaries}

We give some definitions and properties of fractional derivatives and modified generalized Laguerre polynomials.

2.1. The Fractional Derivative in the Caputo Sense. The two most commonly used definitions are the Riemann-Liouville operator and the Caputo operator.
Definition 1. The Riemann-Liouville fractional integral operator of order $\nu(\nu>0)$ is defined as

$$
\begin{aligned}
& J^{v} f(x)=\frac{1}{\Gamma(\nu)} \int_{0}^{x}(x-t)^{\nu-1} f(t) d t, \quad v>0, x>0, \\
& J^{0} f(x)=f(x) .
\end{aligned}
$$

Definition 2. The Caputo fractional derivatives of order $v$ are defined as

$$
\begin{aligned}
D^{\nu} f(x)= & J^{m-v} D^{m} f(x) \\
= & \frac{1}{\Gamma(m-v)} \int_{0}^{x}(x-t)^{m-v-1} \frac{d^{m}}{d t^{m}} f(t) d t, \\
& \quad m-1<v<m, x>0,
\end{aligned}
$$

where $D^{m}$ is the classical differential operator of order $m$.

For the Caputo derivative we have

$$
\begin{gathered}
D^{\nu} C=0, \quad(C \text { is a constant }), \\
D^{\nu} x^{\beta}= \begin{cases}0, & \text { for } \beta \in N_{0} \text { and } \beta<\lceil\nu\rceil, \\
\frac{\Gamma(\beta+1)}{\Gamma(\beta+1-\nu)} x^{\beta-\nu}, & \text { for } \beta \in N_{0} \text { and } \beta \geq\lceil\nu\rceil\end{cases} \\
\quad \text { or } \beta \notin N \text { and } \beta>\lfloor\nu\rfloor,
\end{gathered}
$$

where $\lceil\nu\rceil$ and $\lfloor\nu\rfloor$ are the ceiling and floor functions, respectively, while $N=\{1,2, \ldots\}$ and $N_{0}=\{0,1,2, \ldots\}$.

Caputo's fractional differentiation is a linear operation, similar to the integer-order differentiation

$$
D^{\nu}(\lambda f(x)+\mu g(x))=\lambda D^{\nu} f(x)+\mu D^{v} g(x),
$$

where $\lambda$ and $\mu$ are constants.

2.2. Properties of Modified Generalized Laguerre Polynomials. Let $\Lambda=(0, \infty)$ and let $w^{(\alpha, \beta)}(x)=x^{\alpha} e^{-\beta x}$ be a weight function on $\Lambda$ in the usual sense. Define

$$
L_{w^{(\alpha, \beta)}}^{2}(\Lambda)=\left\{v \mid v \text { is measurable on } \Lambda \text { and }\|v\|_{w^{(\alpha, \beta)}}<\infty\right\}
$$

equipped with the following inner product and norm:

$$
\begin{aligned}
(u, v)_{w^{(\alpha, \beta)}} & =\int_{\Lambda} u(x) v(x) w^{(\alpha, \beta)}(x) d x, \\
\|v\|_{w^{(\alpha, \beta)}} & =(u, v)_{w^{(\alpha, \beta)}}^{1 / 2} .
\end{aligned}
$$

Next, let $L_{i}^{(\alpha, \beta)}(x)$ be the modified generalized Laguerre polynomial of degree $i$ for $\alpha>-1$ and $\beta>0$ is defined by

$$
L_{i}^{(\alpha, \beta)}(x)=\frac{1}{i} x^{-\alpha} e^{\beta x} \partial_{x}^{i}\left(x^{i+\alpha} e^{-\beta x}\right), \quad i=1,2, \ldots
$$


Moreover, for $\alpha>-1$ and $\beta>0$, we have

$$
\begin{aligned}
\partial_{x} L_{i}^{(\alpha, \beta)}(x) & =-\beta L_{i-1}^{(\alpha+1, \beta)}(x), \\
L_{i+1}^{(\alpha, \beta)}(x)= & \frac{1}{i+1}\left[(2 i+\alpha+1-\beta x) L_{i}^{(\alpha, \beta)}(x)\right. \\
& \left.\quad-(i+\alpha) L_{i-1}^{(\alpha, \beta)}(x)\right], \quad i=1,2, \ldots,
\end{aligned}
$$

where $L_{0}^{(\alpha, \beta)}(x)=1$ and $L_{1}^{(\alpha, \beta)}(x)=-\beta x+\Gamma(\alpha+2) / \Gamma(\alpha+1)$.

The set of modified generalized Laguerre polynomials is the $L_{w^{(\alpha, \beta)}}^{2}(\Lambda)$-orthogonal system; namely,

$$
\int_{0}^{\infty} L_{j}^{(\alpha, \beta)}(x) L_{k}^{(\alpha, \beta)}(x) w^{(\alpha, \beta)}(x) d x=h_{k} \delta_{j k}
$$

where $\delta_{j k}$ is the Kronecker function and $h_{k}=\Gamma(k+\alpha+$ 1) $/ \beta^{\alpha+1} k$ !.

The modified generalized Laguerre polynomials of degree $i$ on the interval $\Lambda$ are given by

$$
\begin{array}{r}
L_{i}^{(\alpha, \beta)}(x)=\sum_{k=0}^{i}(-1)^{k} \frac{\Gamma(i+\alpha+1) \beta^{k}}{\Gamma(k+\alpha+1)(i-k) ! k !} x^{k}, \\
i=0,1, \ldots,
\end{array}
$$

where $L_{i}^{(\alpha, \beta)}(0)=\Gamma(i+\alpha+1) / \Gamma(\alpha+1) \Gamma(i+1)$.

The special value

$$
D^{q} L_{i}^{(\alpha, \beta)}(0)=\frac{(-1)^{q} \beta^{q} \Gamma(i+\alpha+1)}{(i-q) ! \Gamma(q+\alpha+1)}, \quad i \geqslant q
$$

will be of important use later.

Since the analytic form of the modified generalized Laguerre polynomials $L_{i}^{(\alpha, \beta)}(x)$ of degree $i$ is given by (11), by using (4), (5), and (11) we get

$$
\begin{aligned}
& D^{\nu} L_{i}^{(\alpha, \beta)}(x) \\
&= \sum_{k=0}^{i}(-1)^{k} \frac{\beta^{k} \Gamma(i+\alpha+1)}{(i-k) ! k ! \Gamma(k+\alpha+1)} D^{\nu} x^{k} \\
&= \sum_{k=\lceil\nu\rceil}^{i}(-1)^{k} \frac{\beta^{k} \Gamma(i+\alpha+1)}{(i-k) ! \Gamma(k-\nu+1) \Gamma(k+\alpha+1)} x^{k-\nu}, \\
& i=\lceil\nu\rceil, \ldots, N .
\end{aligned}
$$

Now, approximating $x^{k-v}$ by $N+1$ terms of modified generalized Laguerre series, we have

$$
x^{k-v}=\sum_{j=0}^{N} b_{j} L_{j}^{(\alpha, \beta)}(x)
$$

where $b_{j}$ is given from (18) with $u(x)=x^{k-\gamma}$, and

$$
b_{j}=\sum_{\ell=0}^{j}(-1)^{\ell} \frac{\beta^{-k+v} j ! \Gamma(k-v+\alpha+\ell+1)}{(j-\ell) !(\ell) ! \Gamma(\ell+\alpha+1)} .
$$

Employing (13)-(15) we get

$$
D^{\nu} L_{i}^{(\alpha, \beta)}(x)=\sum_{j=0}^{N} \Psi_{\nu}(i, j) L_{j}^{(\alpha, \beta)}(x), \quad i=\lceil\nu\rceil, \ldots, N,
$$

where $\Psi_{\nu}(i, j)=\sum_{k=\lceil\nu\rceil}^{i} \theta_{i j k}$, and

$$
\begin{gathered}
\theta_{i j k}=\sum_{\ell=0}^{j}\left((-1)^{k+\ell} \beta^{v} j ! \Gamma(i+\alpha+1) \Gamma(k-v+\alpha+\ell+1)\right) \\
\quad \times((i-k) !(j-\ell) ! \ell ! \Gamma(k-v+1) \\
\quad \times \Gamma(k+\alpha+1) \Gamma(\alpha+\ell+1))^{-1} .
\end{gathered}
$$

A function $u(x) \in L_{w^{(\alpha, \beta)}}^{2}(\Lambda)$ may be expressed in terms of modified generalized Laguerre polynomials as

$$
\begin{aligned}
u(x) & =\sum_{j=0}^{\infty} a_{j} L_{j}^{(\alpha, \beta)}(x), a_{j} \\
& =\frac{1}{h_{k}} \int_{0}^{\infty} u(x) L_{j}^{(\alpha, \beta)}(x) w^{(\alpha, \beta)}(x) d x, \\
& j=0,1,2, \ldots
\end{aligned}
$$

In practice, only the first $(N+1)$ terms of modified generalized Laguerre polynomials are considered. Then we have

$$
u_{N}(x)=\sum_{j=0}^{N} a_{j} L_{j}^{(\alpha, \beta)}(x)=C^{T} \phi(x) .
$$

\subsection{Modified Generalized Laguerre-Gauss and Gauss-Radau} Quadratures. Let $\xi_{G, i, j}^{(\alpha, \beta)}$ and $\xi_{R, i, j}^{(\alpha, \beta)}, 0 \leq j \leq i$, be the zeros of $L_{i+1}^{(\alpha, \beta)}(x)$ and $x \partial_{x} L_{i+1}^{(\alpha, \beta)}(x)$, respectively. They are arranged in ascending order. Denote by $\omega_{Z, i, j}^{(\alpha, \beta)},(0 \leq j \leq i), Z=G, R$, the corresponding Christoffel numbers such that

$$
\int_{\Lambda} \phi(x) w^{(\alpha, \beta)}(x) d x=\sum_{j=0}^{i} \phi\left(\xi_{Z, i, j}^{(\alpha, \beta)}\right) \omega_{Z, i, j}^{(\alpha, \beta)}
$$

where

$$
\omega_{G, i, j}^{(\alpha, \beta)}=\frac{\Gamma(i+\alpha+2)}{\beta^{\alpha} \Gamma(i+2)} \frac{1}{\xi_{G, i, j}^{(\alpha, \beta)}\left[\partial_{x} L_{i+1}^{(\alpha, \beta)}\left(\xi_{G, i, j}^{(\alpha, \beta)}\right)\right]^{2}}, \quad 0 \leq j \leq i .
$$

For the Gauss-Radau weights, we have

$$
\omega_{R, i, j}^{(\alpha, \beta)}= \begin{cases}\frac{(\alpha+1) \Gamma^{2}(\alpha+1) \Gamma(i+1)}{\beta^{\alpha+1} \Gamma(i+\alpha+2)}, & j=0, \\ \frac{\Gamma(i+\alpha+1)}{\beta^{\alpha} \Gamma(i+2)} & 1 \leq j \leq i . \\ \times \frac{1}{L_{i+1}^{(\alpha, \beta)}\left(\xi_{R, i, j}^{(\alpha, \beta)}\right) \partial_{x} L_{i}^{(\alpha, \beta)}\left(\xi_{R, i, j}^{(\alpha, \beta)}\right)}, & 1 \leq\end{cases}
$$


Note that the earlier two types of quadratures have close relations:

$$
\begin{aligned}
& \xi_{R, i, j}^{(\alpha, \beta)}=\xi_{G, i-1, j-1}^{(\alpha+1, \beta)}, \\
& x \omega_{R, i, j}^{(\alpha, \beta)}=\left(\xi_{R, i, j}^{(\alpha, \beta)}\right)^{-1} \omega_{G, i-1, j-1}^{(\alpha+1, \beta)}, \\
& 1 \leq j \leq i .
\end{aligned}
$$

In the next section, we will extend the application of spectral methods (see [37-42]) by using the modified generalized Laguerre polynomials to solve fractional neutral functionaldifferential equations on a semi-infinite domain.

\section{Fractional Neutral \\ Functional-Differential Equations}

In this section, we use the modified generalized Laguerre pseudospectral method based on the modified generalized Laguerre-Gauss quadrature points as the collocation nodes, to solve numerically the following model problem:

$$
\begin{aligned}
& \left(u(x)+a(x) u\left(p_{m} x\right)\right)^{(v)} \\
& \quad=\beta u(x)+\sum_{n=0}^{m-1} b_{n}(x) D^{\gamma_{n}} u\left(p_{n} x\right)+f(x), \quad x \geq 0,
\end{aligned}
$$

with the initial conditions

$$
\sum_{n=0}^{m-1} c_{i n} u^{(n)}(0)=\lambda_{i}, \quad i=0,1, \ldots, m-1 .
$$

Here, $a$ and $b_{n}(n=0,1, \ldots, m-1)$ are given analytical functions; $m-1<v \leq m, 0<\gamma_{0}<\gamma_{1}<\cdots<$ $\gamma_{m-1}<\nu$, and $\beta, p_{n}, c_{i n}, \lambda_{i}$ denote given constants with $0<$ $p_{n}<1(n=0,1, \ldots, m)$. By using the modified generalized Laguerre-Gauss collocation method, we can approximate the fractional neutral functional-differential equations with proportional delays on a semi-infinite domain directly, without any artificial boundary and variable transformation. Let us first introduce some basic notation that will be used in the sequel. We set

$$
S_{N}(0, \infty)=\operatorname{span}\left\{L_{0}^{(\alpha, \beta)}(x), L_{1}^{(\alpha, \beta)}(x), \ldots, L_{N}^{(\alpha, \beta)}(x)\right\},
$$

and we define the discrete inner product and norm as follows:

$$
\begin{aligned}
(u, v)_{w_{N}^{(\alpha, \beta)}} & =\sum_{j=0}^{N} u\left(\xi_{Z, N, j}^{(\alpha, \beta)}\right) v\left(\xi_{Z, N, j}^{(\alpha, \beta)}\right) \omega_{Z, N, j}^{(\alpha, \beta)}, \\
\|u\|_{w_{N}^{(\alpha, \beta)}} & =\sqrt{(u, u)_{w_{N}^{(\alpha, \beta)}}},
\end{aligned}
$$

where $\xi_{Z, N, j}^{(\alpha, \beta)}$ and $\omega_{Z, N, j}^{(\alpha, \beta)}$ are the nodes and the corresponding weights of the modified generalized Laguerre-Gauss quadrature formula on the interval $(0, \infty)$, respectively. Obviously,

$$
(u, v)_{w_{N}^{(\alpha, \beta)}}=(u, v)_{w^{(\alpha, \beta)}}, \quad \forall u, v \in S_{2 N+1} .
$$

Thus, for any $u \in S_{N}(0, \infty)$, the norms $\|u\|_{w_{N}^{(\alpha, \beta)}}$ and $\|u\|_{w^{(\alpha, \beta)}}$ coincide.

Associating with this quadrature rule, we denote by $I_{N}^{L_{T}^{(\alpha, \beta)}}$ the modified generalized Laguerre-Gauss interpolation:

$$
I_{N}^{L_{T}^{(\alpha, \beta)}} u\left(\xi_{Z, N, k}^{(\alpha, \beta)}\right)=u\left(\xi_{Z, N, k}^{(\alpha, \beta)}\right), \quad 0 \leq k \leq N .
$$

The modified generalized Laguerre-Gauss collocation method for solving (24) and (25) is to seek $u_{N}(x) \in S_{N}(0, \infty)$, such that

$$
\begin{gathered}
D^{\nu}\left(u\left(\xi_{Z, N-m, k}^{(\alpha, \beta)}\right)+a\left(\xi_{Z, N-m, k}^{(\alpha, \beta)}\right) u\left(p_{m} \xi_{Z, N-m, k}^{(\alpha, \beta)}\right)\right) \\
=\beta u\left(\xi_{Z, N-m, k}^{(\alpha, \beta)}\right)+\sum_{n=0}^{m-1} b_{n}\left(\xi_{Z, N-m, k}^{(\alpha, \beta)}\right) D^{\gamma_{n}} u\left(p_{n} \xi_{Z, N-m, k}^{(\alpha, \beta)}\right) \\
+f\left(\xi_{Z, N-m, k}^{(\alpha, \beta)}\right), \\
k=0,1, \ldots, N-m, \\
\sum_{n=0}^{m-1} c_{i n} u^{(n)}(0)=\lambda_{i}, \quad i=0,1, \ldots, m-1 .
\end{gathered}
$$

We now derive the algorithm for solving (24) and (25). To do this, let

$$
\begin{gathered}
u_{N}(x)=\sum_{h=0}^{N} a_{h} L_{h}^{(\alpha, \beta)}(x), \\
\mathbf{a}=\left(a_{0}, a_{1}, \ldots, a_{N}\right)^{T} .
\end{gathered}
$$

We first approximate $D^{\nu} u(x)$ and $D^{\gamma_{n}} u(x)$, as (31). By substituting these approximations in (24), we get

$$
\begin{aligned}
& \left(\sum_{h=0}^{N} a_{h} L_{h}^{(\alpha, \beta)}(x)+a(x) \sum_{h=0}^{N} a_{h} L_{h}^{(\alpha, \beta)}\left(p_{m} x\right)\right)^{(\nu)} \\
& =\beta \sum_{h=0}^{N} a_{h} L_{h}^{(\alpha, \beta)}(x) \\
& \quad+\sum_{n=0}^{m-1} \sum_{h=0}^{N} a_{h} b_{n}(x) D^{\gamma_{n}} L_{h}^{(\alpha, \beta)}\left(p_{n} x\right)+f(x) .
\end{aligned}
$$

Making use of (16), we deduce that

$$
\begin{aligned}
& \left(\sum_{h=0}^{N} a_{h} L_{h}^{(\alpha, \beta)}(x)+a(x) \sum_{h=0}^{N} a_{h} L_{h}^{(\alpha, \beta)}\left(p_{m} x\right)\right)^{(v)} \\
& =\beta \sum_{h=0}^{N} a_{h} L_{h}^{(\alpha, \beta)}(x) \\
& \quad+\sum_{n=0}^{m-1} \sum_{h=0}^{N} \sum_{f=0}^{M} a_{h} b_{n}(x) \Psi_{\gamma_{n}}(h, f) L_{f}^{(\alpha, \beta)}\left(p_{n} x\right)+f(x) .
\end{aligned}
$$


Also, by substituting (31) into (25) we obtain

$$
\sum_{n=0}^{m-1} \sum_{f=0}^{M} a_{i n} D^{(n)} L_{f}^{(\alpha, \beta)}(0)=\lambda_{i}
$$

Now, collocating (33) at the $(N-m+1)$ modified generalized Laguerre-Gauss interpolation points yields

$$
\begin{aligned}
& \left(\sum_{h=0}^{N} a_{h} L_{h}^{(\alpha, \beta)}\left(\xi_{Z, N-m, k}^{(\alpha, \beta)}\right)\right. \\
& \left.+a\left(\xi_{Z, N-m, k}^{(\alpha, \beta)}\right) \sum_{h=0}^{N} a_{h} L_{h}^{(\alpha, \beta)}\left(p_{m} \xi_{Z, N-m, k}^{(\alpha, \beta)}\right)\right)^{(v)} \\
& =\beta \sum_{h=0}^{N} a_{h} L_{h}^{(\alpha, \beta)}\left(\xi_{Z, N-m, k}^{(\alpha, \beta)}\right) \\
& \quad+\sum_{n=0}^{m-1} \sum_{h=0}^{N} \sum_{f=0}^{M} a_{h} b_{n}\left(\xi_{Z, N-m, k}^{(\alpha, \beta)}\right) \Psi_{\gamma_{n}}(h, f) L_{f}^{(\alpha, \beta)} \\
& \times\left(p_{n} \xi_{Z, N-m, k}^{(\alpha, \beta)}\right)+f\left(\xi_{Z, N-m, k}^{(\alpha, \beta)}\right) .
\end{aligned}
$$

Next (34), after using (12), can be written as

$$
\sum_{n=0}^{m-1} \sum_{f=0}^{M}(-1)^{q} a_{i n} \frac{\beta^{q} \Gamma(f+\alpha+1)}{(f-q) ! \Gamma(q+\alpha+1)}=\lambda_{i} .
$$

Finally, (35) with relation $(36)$ generates $(N+1)$ set of algebraic equations which can be solved for the unknown coefficients $a_{j}, j=0,1,2, \ldots, N$, by using any standard solver technique.

\section{Numerical Results}

In this section, we implement the proposed method to solve two numerical examples from the fractional neutral functional-differential equations with proportional delays in semi-infinite intervals to demonstrate the accuracy and capability of the proposed algorithm. The absolute errors in the given tables are the values of $\left|u(x)-u_{N}(x)\right|$ at selected points.

Example 1. Consider the following fractional neutral functional-differential equation with proportional delay:

$$
\begin{gathered}
u^{1 / 2}(x)=-u(x)+\frac{1}{3} u\left(\frac{x}{4}\right)+\frac{1}{2} u^{1 / 2}\left(\frac{x}{4}\right)+g(x), \\
u(0)=1, \\
x \in[0,5],
\end{gathered}
$$

where

$$
\begin{aligned}
g(x)= & -\frac{1}{\Gamma(1 / 2)} \int_{0}^{x}(x-t)^{-1 / 2} \sin t d t+\cos x-\frac{1}{3} \cos \left(\frac{x}{4}\right) \\
& +\frac{1}{2 \Gamma(1 / 2)} \int_{0}^{x}(x-t)^{-1 / 2} \sin \left(\frac{t}{4}\right) d t
\end{aligned}
$$

and the exact solution is given by $u(x)=\cos x$.
TABLE 1: Absolute errors using MGLC method at $N=22$ for Example 1.

\begin{tabular}{lccc}
\hline$x$ & $\alpha=1, \beta=2$ & $\alpha=4, \beta=3$ & $\alpha=\beta=5$ \\
\hline 0.0 & $7.993 \cdot 10^{-15}$ & $0.000 \cdot 10^{-00}$ & $2.220 \cdot 10^{-16}$ \\
0.5 & $5.164 \cdot 10^{-4}$ & $7.041 \cdot 10^{-4}$ & $1.746 \cdot 10^{-4}$ \\
1.0 & $5.066 \cdot 10^{-4}$ & $5.763 \cdot 10^{-4}$ & $1.416 \cdot 10^{-4}$ \\
1.5 & $3.521 \cdot 10^{-4}$ & $4.936 \cdot 10^{-4}$ & $1.257 \cdot 10^{-4}$ \\
2.0 & $2.793 \cdot 10^{-4}$ & $4.268 \cdot 10^{-4}$ & $1.059 \cdot 10^{-4}$ \\
2.5 & $4.480 \cdot 10^{-4}$ & $3.862 \cdot 10^{-4}$ & $8.856 \cdot 10^{-5}$ \\
3.0 & $2.269 \cdot 10^{-4}$ & $3.437 \cdot 10^{-4}$ & $8.429 \cdot 10^{-5}$ \\
3.5 & $1.998 \cdot 10^{-4}$ & $3.113 \cdot 10^{-4}$ & $7.249 \cdot 10^{-5}$ \\
4.0 & $5.164 \cdot 10^{-4}$ & $3.037 \cdot 10^{-4}$ & $6.271 \cdot 10^{-5}$ \\
4.5 & $1.141 \cdot 10^{-3}$ & $2.841 \cdot 10^{-4}$ & $1.159 \cdot 10^{-4}$ \\
5.0 & $1.154 \cdot 10^{-3}$ & $2.268 \cdot 10^{-4}$ & $5.200 \cdot 10^{-5}$ \\
\hline
\end{tabular}

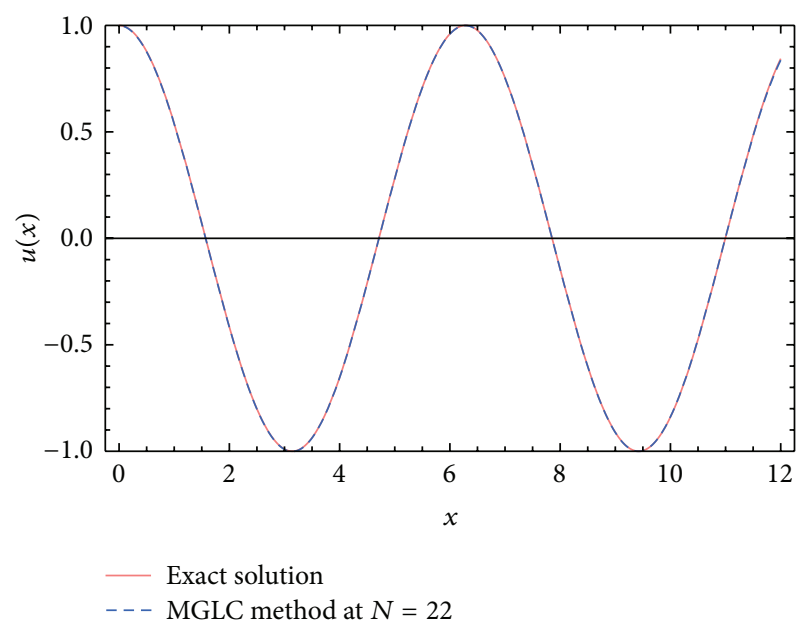

FIGURE 1: Graph of exact solution and approximate solution for $\alpha=$ $4, \beta=2$ at $N=22$ for Example 1 .

Table 1 lists the results obtained by the modified generalized Laguerre collocation method in terms of maximum absolute errors at $N=22$ with $\alpha=1, \beta=2, \alpha=4, \beta=3$, and $\alpha=\beta=5$. In the case of $\alpha=4, \beta=2$, the approximate solution by the presented method is shown in Figure 1, to make it easier to compare with the exact solution.

Example 2. Consider the following fractional neutral functional-differential equation with proportional delay:

$$
\begin{aligned}
u^{5 / 2}(x)= & u(x)+u^{1 / 2}\left(\frac{x}{2}\right)+u^{3 / 2}\left(\frac{x}{3}\right)+\frac{1}{2} u^{5 / 2}\left(\frac{x}{4}\right) \\
& +\frac{\Gamma(4)}{\Gamma(3 / 2)} x^{1 / 2}-x^{3}-\frac{\Gamma(4)}{\Gamma(7 / 2)}\left(\frac{x}{2}\right)^{1 / 2} \\
& -\frac{\Gamma(4)}{\Gamma(5 / 2)}\left(\frac{x}{3}\right)^{3 / 2}-\frac{\Gamma(4)}{2 \Gamma(3 / 2)}\left(\frac{x}{4}\right)^{1 / 2}, \quad x \in[0,1],
\end{aligned}
$$




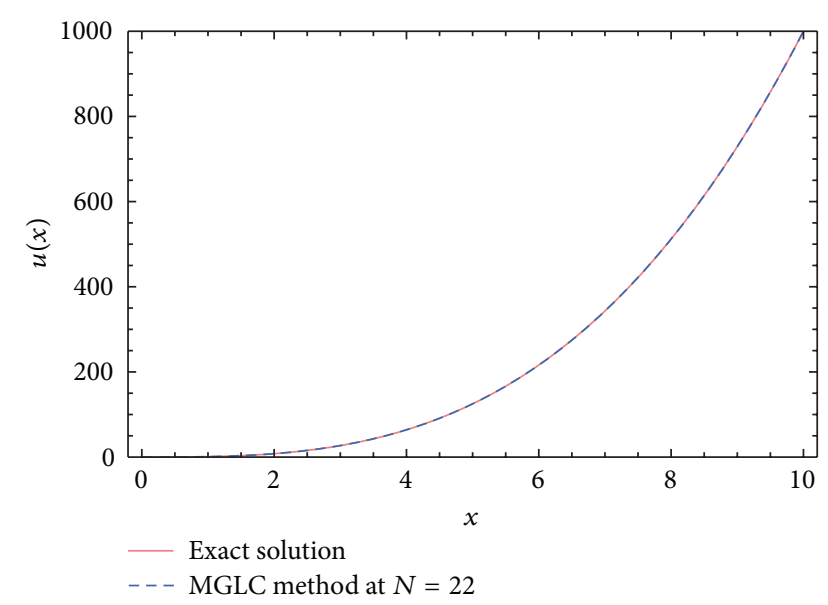

FIGURE 2: Graph of exact solution and approximate solution for $\alpha=$ 2, $\beta=1$ at $N=22$ for Example 2 .

TABle 2: Absolute errors using MGLC method at $N=22$ for Example 2.

\begin{tabular}{lccc}
\hline$x$ & $\alpha=0, \beta=1$ & $\alpha=3, \beta=2$ & $\alpha=\beta=2$ \\
\hline 0.0 & $3.221 \cdot 10^{-11}$ & $5.995 \cdot 10^{-15}$ & $1.154 \cdot 10^{-14}$ \\
0.1 & $7.800 \cdot 10^{-5}$ & $2.466 \cdot 10^{-5}$ & $1.057 \cdot 10^{-5}$ \\
0.2 & $3.118 \cdot 10^{-4}$ & $1.117 \cdot 10^{-4}$ & $1.067 \cdot 10^{-5}$ \\
0.3 & $4.312 \cdot 10^{-4}$ & $2.276 \cdot 10^{-4}$ & $8.451 \cdot 10^{-5}$ \\
0.4 & $2.384 \cdot 10^{-4}$ & $3.035 \cdot 10^{-4}$ & $3.128 \cdot 10^{-4}$ \\
0.5 & $2.027 \cdot 10^{-4}$ & $3.301 \cdot 10^{-4}$ & $6.556 \cdot 10^{-4}$ \\
0.6 & $6.146 \cdot 10^{-4}$ & $3.251 \cdot 10^{-4}$ & $1.075 \cdot 10^{-3}$ \\
0.7 & $6.439 \cdot 10^{-4}$ & $3.181 \cdot 10^{-4}$ & $1.551 \cdot 10^{-3}$ \\
0.8 & $4.964 \cdot 10^{-6}$ & $3.332 \cdot 10^{-4}$ & $2.096 \cdot 10^{-3}$ \\
0.9 & $1.417 \cdot 10^{-3}$ & $3.789 \cdot 10^{-4}$ & $2.756 \cdot 10^{-3}$ \\
1.0 & $3.521 \cdot 10^{-3}$ & $4.465 \cdot 10^{-4}$ & $3.593 \cdot 10^{-3}$ \\
\hline
\end{tabular}

subject to

$$
\begin{gathered}
u(0)=0, \\
u^{\prime}(0)=0, \\
u^{\prime \prime}(0)=0,
\end{gathered}
$$

with exact solution $u(x)=x^{3}$.

In Table 2, we list the absolute errors obtained by the modified generalized Laguerre collocation method, with several values of $\alpha, \beta$ and at $N=22$. Meanwhile, Figure 2 presents the MGLC solution with $\alpha=2, \beta=1$ at $N=22$ and exact solution, which are found to be in excellent agreement.

\section{Conclusions}

We have presented the modified generalized LaguerreGauss collocation scheme for approximating the solution of fractional neutral functional-differential equations with proportional delays on the half-line. The technique was based on modified generalized Laguerre polynomials and Gauss quadrature integration of such polynomials, which is defined on the half-line. In the previous section, two numerical results were given to demonstrate the accuracy, efficiency, and versatility of the proposed method on the half-line. Indeed, reasonable numerical results were achieved by choosing few modified generalized Laguerre-Gauss collocation points.

\section{Conflict of Interests}

The authors declare that there is no conflict of interests regarding the publication of this paper.

\section{Acknowledgment}

This paper was funded by the Deanship of Scientific Research (DSR) at King Abdulaziz University, Jeddah, under Grant no. (4-135-35-RG). The authors, therefore, acknowledge DSR technical and financial support.

\section{References}

[1] S. Samko, A. A. Kilbas, and O. I. Marichev, Fractional Integrals and Derivatives-Theory and Applications, Gordon and Breach, Linghorne, Pa, USA, 1993.

[2] D. Baleanu, K. Diethelm, E. Scalas, and J. J. Trujillo, Fractional Calculus, vol. 3 of Series on Complexity, Nonlinearity and Chaos, World Scientific, 2012.

[3] A. M. O. Anwar, F. Jarad, D. Baleanu, and F. Ayaz, "Fractional Caputo heat equation within the double Laplace transform," Romanian Journal of Physics, vol. 58, no. 1-2, pp. 15-22, 2013.

[4] A. H. Bhrawy and M. Alghamdi, "A shifted Jacobi-GaussLobatto collocation method for solving nonlinear fractional Langevin equation involving two fractional orders in different intervals," Boundary Value Problems, vol. 2012, article 62, 2012.

[5] A. Kadem and D. Baleanu, "On fractional coupled WhithamBroer-Kaup equations," Romanian Journal of Physics, vol. 56, no. 5-6, pp. 629-635, 2011.

[6] M. Ortigueira, "Introduction to fraction linear systems-part 1: continuous-time case," IEE Proceedings Vision, Image, Signal Processing, vol. 147, pp. 6-70, 2000.

[7] M.-B. Wei and D.-Q. Zeng, "Fractional perturbation technique of fractional differentiable functions," Romanian Journal of Physics, vol. 57, no. 9-10, pp. 1278-1284, 2012.

[8] S. J. Sadati, R. Ghaderi, and A. Ranjbar, "Some fractional comparison results and stabliity theorem for time delay systems," Romanian Reports in Physics, vol. 65, pp. 94-102, 2013.

[9] F. Gao, X. Lee, H. Tong, F. Fei, and H. Zhao, "Identification of unknown parameters and orders via cuckoo search oriented statistically by differential evolution for noncommensu- rate fractional-order chaotic systems," Abstract and Applied Analysis, vol. 2013, Article ID 382834, 19 pages, 2013.

[10] F. Gao, X. Lee, F. Fei, H. Tong, Y. Deng, and H. Zhao, "Identification time-delayed fractional order chaos with functional extrema model via differential evolution," Expert Systems with Applications, vol. 41, pp. 1601-1608, 2014.

[11] A. H. Bhrawy, D. Baleanu, and L. M. Assas, "Efficient generalized Laguerre-spectral methods for solving multi-term fractional differential equations on the half line," Journal of Vibration and Control, vol. 20, no. 7, pp. 973-985, 2014. 
[12] D. Baleanu, A. H. Bhrawy, and T. M. Taha, "Two efficient generalized Laguerre spectral algorithms for fractional initial value problems," Abstract and Applied Analysis, vol. 2013, Article ID 546502, 10 pages, 2013.

[13] J. Deng and Z. Deng, "Existence of solutions of initial value problems for non-linear fractional differential equations," Applied Mathematics Letters, vol. 32, pp. 6-12, 2014.

[14] Y. Zhao, D.-F. Cheng, and X.-J. Yang, "Approximation solutions for local fractional Schrödinger equation in the onedimensional Cantorian system," Advances in Mathematical Physics, vol. 2013, Article ID 291386, 5 pages, 2013.

[15] M. H. Heydari, M. R. Hooshmandasl, F. M. Maalek Ghaini, and M. Li, "Chebyshev wavelets method for solution of nonlinear fractional integrodifferential equations in a large interval," Advances in Mathematical Physics, vol. 2013, Article ID 482083, 12 pages, 2013.

[16] Y. Z. Povstenko, "Fundamental solutions to time-fractional advection diffusion equation in a case of two space variables," Mathematical Problems in Engineering, vol. 2014, Article ID 705364, 7 pages, 2014.

[17] B. Yu and X. Jiang, "A fractional anomalous diffusion model and numerical simulation for sodium ion transport in the intestinal wall," Advances in Mathematical Physics, vol. 2013, Article ID 479634, 8 pages, 2013.

[18] Y. Zhao, A. Xiao, L. Li, and C. Zhang, "Variational iteration method for singular perturbation initial value problems with delays," Mathematical Problems in Engineering, vol. 2014, Article ID 850343, 8 pages, 2014.

[19] F. X. Sun, C. Liu, and Y. M. Cheng, "An improved interpolating element-free Galerkin method based on nonsingular weight functions," Mathematical Problems in Engineering, vol. 2014, Article ID 323945, 13 pages, 2014.

[20] D. Rostamy, M. Alipour, H. Jafari, and D. Baleanu, "Solving multi-term orders fractional matrices of Bps with convergence analysis," Romanian Reports in Physics, vol. 65, pp. 334-349, 2013.

[21] J. J. R. Garcia, M. G. Calderon, J. M. Ortiz, and D. Baleanu, "Motion of a particle in a resisting medium using fractional calculus approach," Proceedings of the Romanian Academy A: Mathematics, Physics, Technical Sciences, Information Science, vol. 14, no. 1, pp. 42-47, 2013.

[22] E. Ishiwata and Y. Muroya, "Rational approximation method for delay differential equations with proportional delay," Applied Mathematics and Computation, vol. 187, no. 2, pp. 741-747, 2007.

[23] X. Chen and L. Wang, "The variational iteration method for solving a neutral functional-differential equation with proportional delays," Computers \& Mathematics with Applications, vol. 59, no. 8, pp. 2696-2702, 2010.

[24] M. Heydari, G. B. Loghmani, and S. M. Hosseini, "Operational matrices of Chebyshev cardinal functions and their application for solving delay differential equations arising in electrodynamics with error estimation," Applied Mathematical Modelling, vol. 37, no. 14-15, pp. 7789-7809, 2013.

[25] E. Tohidi, A. H. Bhrawy, and K. Erfani, "A collocation method based on Bernoulli operational matrix for numerical solution of generalized pantograph equation," Applied Mathematical Modelling, vol. 37, no. 6, pp. 4283-4294, 2013.

[26] E. H. Doha, A. H. Bhrawy, D. Baleanu, and R. M. Hafez, "A new Jacobi rational-Gauss collocation method for numerical solution of generalized pantograph equations," Applied Numerical Mathematics, vol. 77, pp. 43-54, 2014.
[27] M. P. Tripathi, V. K. Baranwal, R. K. Pandey, and O. P. Singh, "A new numerical algorithm to solve fractional differential equations based on operational matrix of generalized hat functions," Communications in Nonlinear Science and Numerical Simulation, vol. 18, no. 6, pp. 1327-1340, 2013.

[28] A. H. Bhrawy and M. M. Al-Shomrani, "A shifted Legendre spectral method for fractional-order multi-point boundary value problems," Advances in Difference Equations, vol. 2012, article 8, 2012.

[29] X. Ma and C. Huang, "Spectral collocation method for linear fractional integro-differential equations," Applied Mathematical Modelling, vol. 38, no. 4, pp. 1434-1448, 2014.

[30] Y. Yang and Y. Huang, "Spectral-collocation methods for fractional pantograph delay-integrodifferential equations," Advances in Mathematical Physics, vol. 2013, Article ID 821327, 14 pages, 2013.

[31] F. Yin, J. Song, Y. Wu, and L. Zhang, "Numerical solution of the fractional partial differential equations by the twodimensional fractional-order Legendre functions," Abstract and Applied Analysis, vol. 2013, Article ID 562140, 13 pages, 2013.

[32] F. Yin, J. Song, H. Leng, and F. Lu, "Couple of the variational iteration method and fractional-order Legendre functions method for fractional differential equations," The Scientific World Journal, vol. 2014, Article ID 928765, 9 pages, 2014.

[33] M. Kamrani, "Numerical solution of stochastic fractional differential equations," Numerical Algorithms, 2014.

[34] A. H. Bhrawy and M. A. Alghamdi, "The operational matrix of Caputo fractional derivatives of modified generalized Laguerre polynomials and its applications," Advances in Difference Equations, vol. 2013, article 307, 2013.

[35] D. Baleanu, A. H. Bhrawy, and T. M. Taha, "A modified generalized Laguerre spectral method for fractional differential equations on the half line," Abstract and Applied Analysis, vol. 2013, Article ID 413529, 12 pages, 2013.

[36] A. H. Bhrawy, M. M. Alghamdi, and T. M. Taha, "A new modified generalized Laguerre operational matrix of fractional integration for solving fractional differential equations on the half line," Advances in Difference Equations, vol. 2012, article 179, 2012.

[37] C. Canuto, M. Y. Hussaini, A. Quarteroni, and T. A. Zang, Spectral Methods in Fluid Dynamics, Springer, New York, NY, USA, 1988.

[38] B. Fornberg, A practical Guide to Pseudospectral Methods, Cambridge University Press, Cambridge, mass, USA, 1996.

[39] E. H. Doha, A. H. Bhrawy, and R. M. Hafez, "On shifted Jacobi spectral method for high-order multi-point boundary value problems," Communications in Nonlinear Science and Numerical Simulation, vol. 17, no. 10, pp. 3802-3810, 2012.

[40] E. H. Doha and A. H. Bhrawy, "An efficient direct solver for multidimensional elliptic Robin boundary value problems using a Legendre spectral-Galerkin method," Computers \& Mathematics with Applications, vol. 64, no. 4, pp. 558-571, 2012.

[41] R. Peyret, Spectral Methods for Incompressible Viscous Flow, Springer, New York, NY, USA, 2002.

[42] A. H. Bhrawy, "A Jacobi-Gauss-Lobatto collocation method for solving generalized Fitzhugh-Nagumo equation with timedependent coefficients," Applied Mathematics and Computation, vol. 222, pp. 255-264, 2013. 


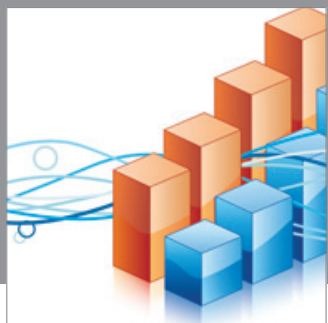

Advances in

Operations Research

mansans

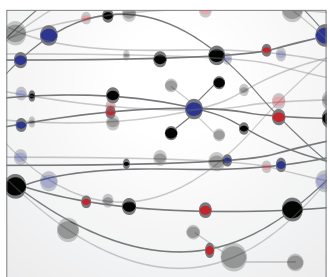

The Scientific World Journal
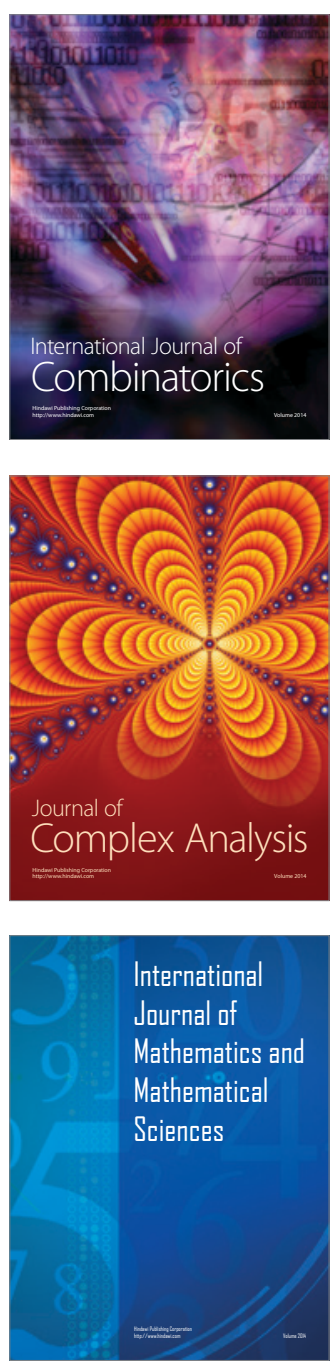
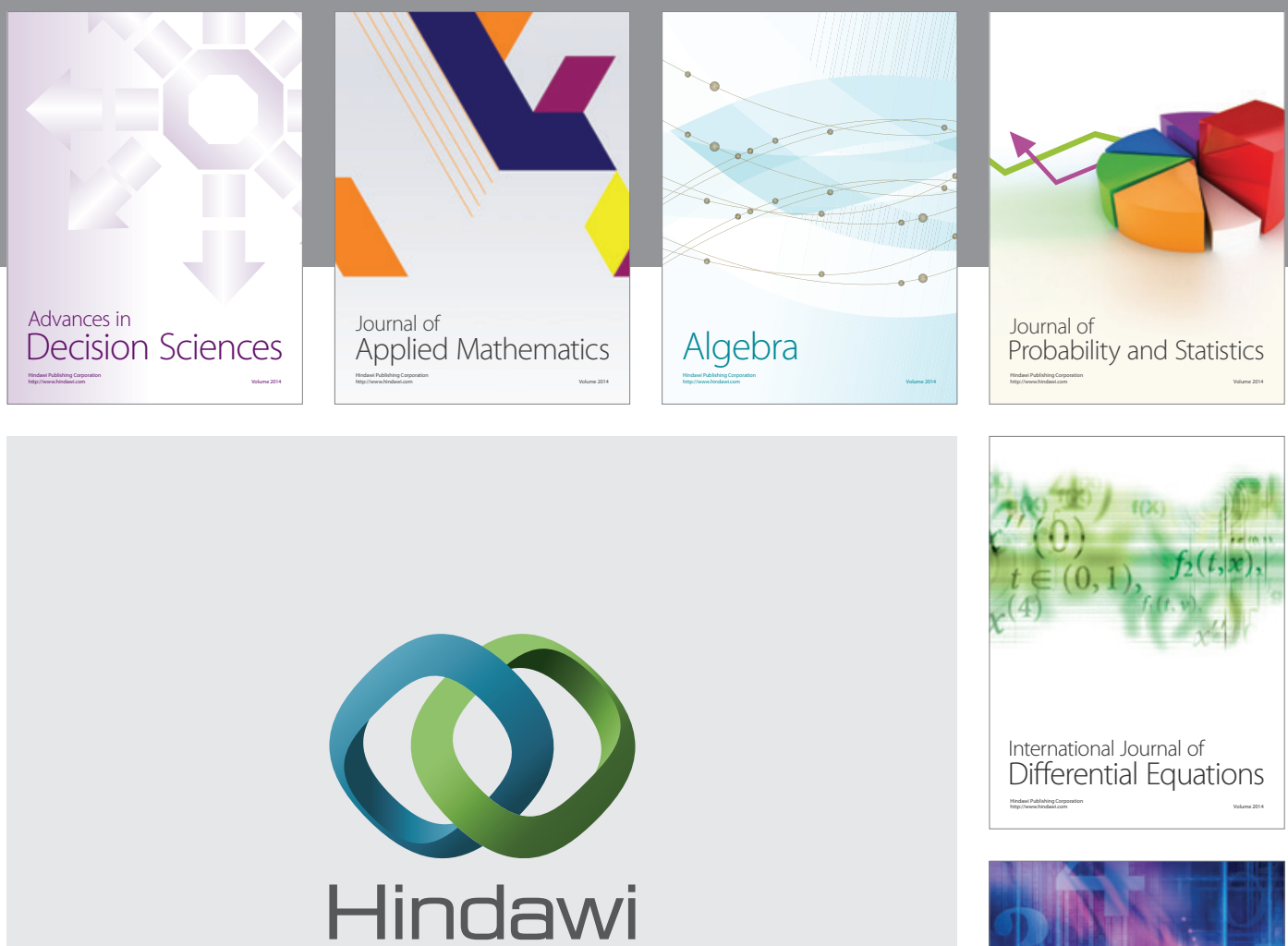

Submit your manuscripts at http://www.hindawi.com
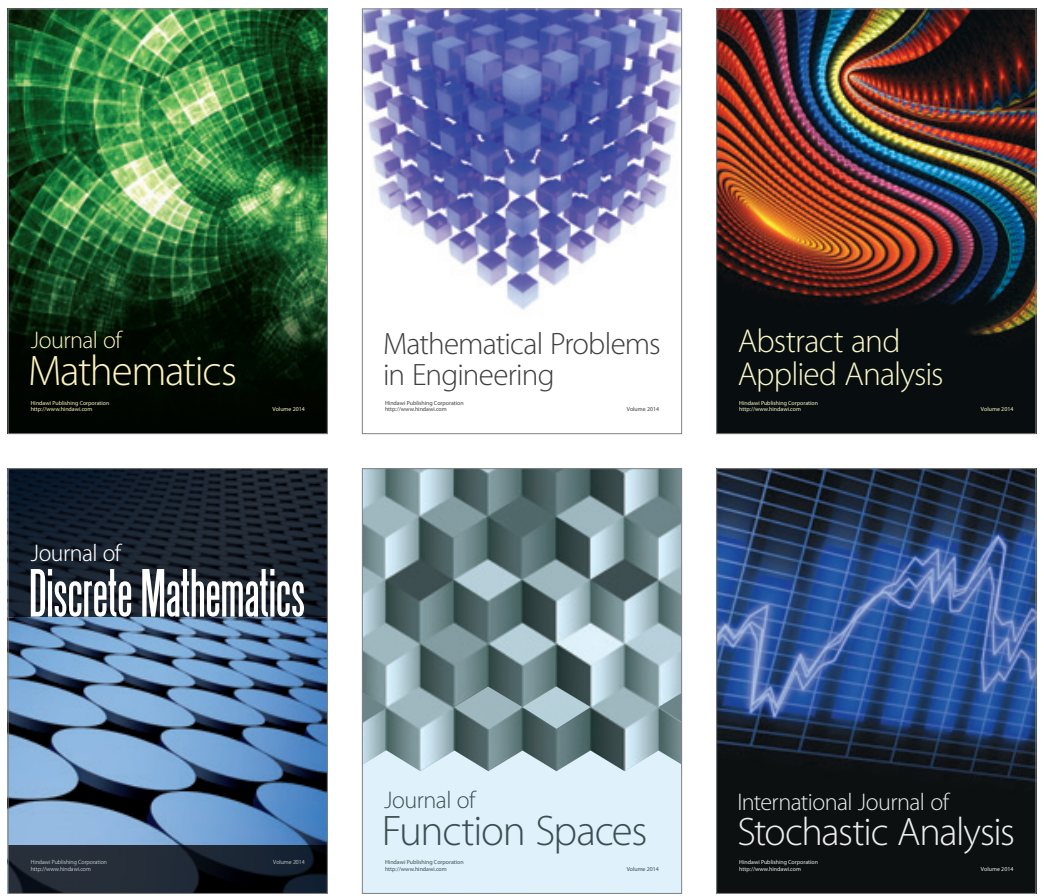

Journal of

Function Spaces

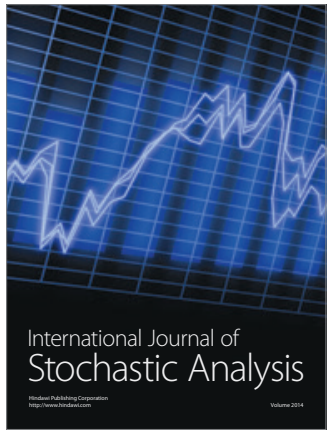

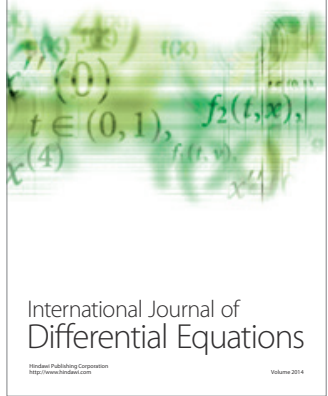
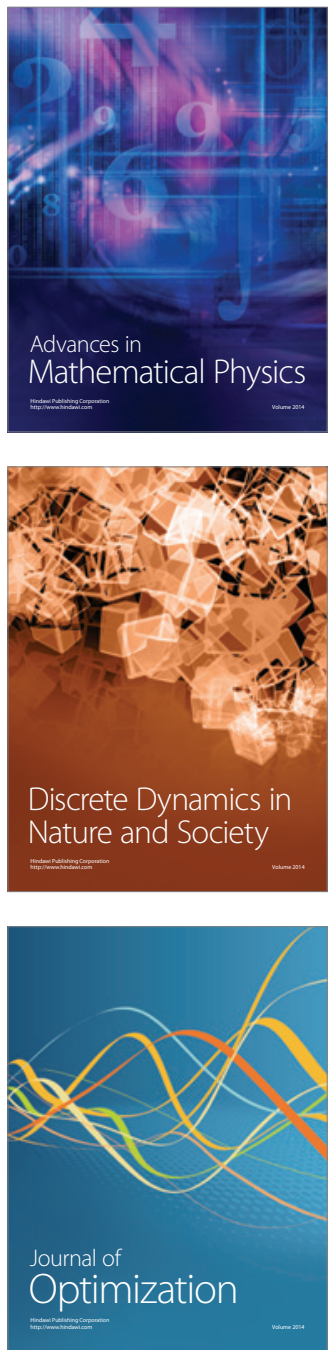